\title{
A simplified version of the Walsh clinical prediction rule was accurate for detecting streptococcal pharyngitis
}

\author{
McGinn TG, Deluca J, Ahlawat SK, et al. Validation and modification of streptococcal pharyngitis clinical prediction rules. \\ Mayo Clin Proc 2003;78:289-93. \\ QUESTION: In adults with symptoms of upper respiratory tract infection or sore throat, \\ what is the accuracy of a simplified version of the Walsh clinical prediction rule (CPR) \\ for detecting group $A \beta$ haemolytic streptococcal (GABHS) pharyngitis?
}

\section{Design}

A cohort study to validate a simplified version of a previously derived prediction rule.

\section{Setting}

Primary care clinic of an inner city hospital in New York City, New York, USA.

Source of funding. Albert Einstein College of Medicine, Montefiore Medical Center.

For correspondence Dr T G McGinn, Mount Sinai School of Medicine, New York NY, USA.

thomas.mcginn@ mountsinai.org

\section{Patients}

171 consecutive walk in patients (mean age 35 y, 77\% women) with symptoms of upper respiratory tract infection or sore throat.

Description of prediction guide

All patients were assessed using 5 clinical predictors. The simplified and original Walsh CPRs used similar clinical predictors but different scores: oral temperature (original: 3 points for each degree $>36.1^{\circ} \mathrm{C}$; simplified: 1 point for

Likelihood ratios for GABHS pharyngitis according to derivation v validation*

\begin{tabular}{lll}
$\begin{array}{l}\text { Score } \\
\text { Derivation (original scoring) }\end{array}$ & $\begin{array}{l}\text { Percentage of patients with } \\
\text { GABHS pharyngitis (95\% CI) }\end{array}$ \\
\hline-10 to 0 & 0.16 & $5 \%(0$ to 12$)$ \\
\hline 1 to 10 & 1.12 & $26 \%(13$ to 41$)$ \\
\hline 11 to 20 & 1.25 & $28 \%(14$ to 45$)$ \\
\hline 21 to 30 & 9.77 & $75 \%(48$ to 93$)$ \\
\hline 31 to 40 & 9.74 & $75 \%(19$ to 99$)$ \\
\hline Validation (simplified scoring) & & \\
\hline-1 & 0.16 & $5 \%(0$ to 12$)$ \\
\hline 0 & 0.62 & $16 \%(7$ to 30$)$ \\
\hline 1 & 2.61 & $44 \%(28$ to 62$)$ \\
\hline 2 & 4.25 & $57 \%(29$ to 82$)$ \\
\hline 3 & 16.23 & $83 \%(36$ to 100$)$ \\
\hline
\end{tabular}

*Abbreviations defined in glossary. $>38.3^{\circ} \mathrm{C}$ ), close contact with a person believed to have a streptococcal infection (original: 17 points; simplified: 1 point), cough (original: -7 points; simplified: -1 point), presence or absence of pharyngeal or tonsillar exudates (original: 6 points; simplified: 1 point), and enlarged or tender anterior cervical lymph nodes (original: 11 points; simplified: 1 point). The scoring of the simplified Walsh CPR was modified from the original scoring system used by physicians in their day to day practice, in which they give equal weight to each of the 5 predictors. All patients had throat cultures taken for GABHS pharyngitis.

\section{Main outcome measures}

Patient scores and likelihood ratios on the 5 clinical predictors to determine risk of GABHS. The simplified Walsh CPR was scored by giving equal weight of 1 point for all clinical predictors except for the presence of cough, which was assigned a -1 weight because of its association with the absence of GABHS infection. Likelihood ratios and area under the receiver operating characteristic (ROC) curve were calculated for points determining risk of streptococcal pharyngitis.

\section{Main results}

39 patients $(24 \%)$ had GABHS pharyngitis. Both the original Walsh CPR and the simplified version of the Walsh CPR accurately predicted the probability of a positive culture for GABHS pharyngitis (ROC $0.71 v$ $0.81, \mathrm{p}=0.21$ ) (table).

\section{Conclusion}

In adults with symptoms of upper respiratory tract infection or sore throat, the simplified version of the Walsh clinical prediction rule was accurate for detecting the presence of group A $\beta$ haemolytic streptococcal pharyngitis.

*See glossary.

\section{COMMENTARY}

Although most sore throats lead only to temporary illness, streptococcal pharyngitis may have prolonged symptoms and sometimes local suppurative and serious immune complications. Antibiotic treatment decreases symptoms, reduces transmission, and reduces suppurative complications and acute rheumatic fever. Since no rapid test can perfectly identify streptococcal pharyngitis, management decisions for patients with a sore throat may be made according to the probability that they have streptococcal pharyngitis. ${ }^{1}$

The study by McGinn $e$ al adds to the growing evidence that the probability of having streptococcal pharyngitis can be judged based on a few key signs and symptoms. The simple algorithm used by McGinn $e t$ al, which counted points ( 1 each for fever, pharyngeal or tonsillar exudates, adenopathy, and exposure to streptococcal pharyngitis, and a negative point for cough), accurately estimated disease probability according to the number of points present. This diagnostic rule was similar to those published by Centor et al (which omitted exposure to streptococcal pharyngitis as a variable) ${ }^{2}$ and by McIsaac $e t$ al (which omitted exposure, but included age) ${ }^{3}$, which were both independently validated.

Thus, for a given patient, the presence of each variable (fever, exudates, adenopathy, and lack of cough) independently increases the likelihood of streptococcal pharyngitis. Adjustment for the local prevalence of streptococcal pharyngitis can then lead to an estimate of disease probability for the individual patient that is accurate enough to guide management decisions.

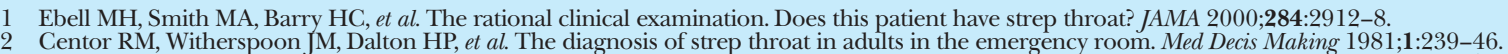

Centor RM, Witherspoon JM, Dalton HP, et al. The diagnosis of strep throat in adults in the emergency room. Med Decis Making 1981;1:239-46.
McIsaac WJ, White D, Tannenbaum D, et al. A clinical score to reduce unnecessary antibiotic use in patients with sore throat. CMAJ 1998;158:75-83. McIsaac WJ, White D, Tannenbaum D, et al. A clinical score to reduce unnecessary antibiotic use in patients with sore throat. CMAJ 1998; 158:75-83.
Poses RM, Cebul RD, Collins M, et al. The importance of disease prevalence in transporting clinical prediction rules. The case of streptococcal pharyngitis. Ann Intern 